\title{
Influence de la densité d'élevage sur la croissance et les caractéristiques de carcasse de poulets élevés au sol
}

\author{
F.H. RICARD \\ avec la collaboration technique de G. MARCHE \\ INRA, Station de Recherches avicoles, \\ Centre de Recherches de Tours, Nouzilly, F 37380 Monnaie
}

\section{Résumé}

Des poulets mâles et femelles d'un croisement Cornish $\times$ White Rock ont été élevés au sol avec une densité forte ou faible, respectivement 23 et 10 sujets par mètre carré avec une même longueur de mangeoire par sujet. Les autres modalités d'élevage étaient strictement identiques.

Les animaux du lot à forte densité avaient une vitesse de croissance et un angle de poitrine significativement plus faibles que ceux du lot à densité faible. Quand la vitesse de croissance était maintenue constante, la dissection des carcasses a montré que le lot à forte densité donnait des poulets significativement plus gras avec un moins bon rendement en viande. Ces poulets présentaient également un pourcentage de foie plus élevé et un pourcentage de cou plus faible que ceux du lot à densité faible.

La comparaison des sexes a confirmé que les poulettes, plus légères, présentaient un état d'engraissement plus élevé que les coquelets. Chez ces derniers, le pourcentage des pattes, de la tête et de l'ensemble cuisses + pilons était plus élevé et le pourcentage des muscles pectoraux plus faible.

Mots clés: Densité d'élevage, sexe, poulet, croissance, angle de poitrine, engraissement, composition anatomique des carcasses.

\section{Introduction}

L'un des facteurs de l'intensification de la production avicole est l'augmentation des densités d'élevage puisqu'on peut ainsi produire une plus grande quantité de viande par mètre carré de poulailler. Au plan zootechnique, la vitesse de croissance est diminuée mais, généralement, l'indice de consommation et le taux de mortalité ne sont pas affectés (nombreuses références dont celles de BuckLAND et al,, 1971 ; LoPEz \& Escalante, 1982 ; Polanco \& Lopez, 1984 ; Proudfoot \& Hulan, 1985 pour les élevages au sol ; Chrappa et al., 1982 ou Scholtyssek \& Gschwindt-Ensinger, 1983 pour les élevages de poulets en cage).

L'influence de la densité sur la qualité des carcasses a été moins souvent étudiée. On a pu observer qu'une densité faible s'accompagnait d'un plus fort pourcentage de carcasses classées en première catégorie (Moreng et al., 1961 ; BuCKLAND et al., 1971 ; 
Proudfoot, 1973) et d'une fréquence plus faible d'ampoules au bréchet (STANley \& Krueger, 1981). D'après les travaux de Proudfoot (1973) et ceux de Scholtyssek (1973), le rendement à l'abattage ne semble pas modifié. En ce qui concerne l'état d'engraissement, les différences ne sont pas significatives pour WEAVER et al. (1982), mais pour Vo \& FANGUY (1982), les dépôts gras abdominaux augmentent quand la densité s'élève. Enfin, Scholtyssek (1973) a observé un meilleur pourcentage des morceaux nobles (poitrine et cuisses) quand la densité est faible, tandis que ce pourcentage varie peu dans le travail de Tsonkov \& BerEmski (1975), même s'il a tendance à diminuer quand la densité augmente.

Dans le cadre d'un travail plus général sur les facteurs de variation de la qualité de carcasse du poulet, nous avons comparé deux densités : la première, 10 sujets au mètre carré, correspond à un élevage traditionnel ; la seconde, 23 au mètre carré, correspond aux élevages intensifs modernes. Quelques-uns des résultats obtenus ont été présentés à la $7^{\mathrm{e}}$ Conférence Avicole Européenne de Paris (RICARD et al., 1986). En particulier, les deux lots ne présentaient pas de différence significative pour les caractéristiques organoleptiques. Nous indiquons dans le présent article l'ensemble des résultats obtenus pour la composition anatomique des carcasses des deux sexes. Ces résultats permettent de compléter les données, souvent peu précises, relevées dans la bibliographie et, secondairement, d'avoir des chiffres comparatifs pour la composition anatomique des coquelets et des poulettes.

\section{Matériel et méthodes}

\section{A. Elevage des animaux}

Les poussins utilisés ont été obtenus en mars 1983 à la Station de Recherches avicoles à partir d'un croisement entre des coqs de type Cornish et des poules de type White-Rock. Ils ont été mis en place le même jour dans deux cases contiguës de la même poussinière d'une superficie de $16 \mathrm{~m}^{2}$ chacune. La première a reçu 157 sujets des 2 sexes, soit 10 par mètre carré, et la seconde 367 sujets, soit 23 par mètre carré. Dans cette dernière case, la longueur des mangeoires était doublée de façon à éviter un effet éventuel lié à la compétition alimentaire.

Toutes les autres modalités d'élevage étaient identiques. La litière était constituée de copeaux de bois. Après 4 jours d'éclairement continu, les animaux étaient soumis à un nycthémère constant $12: 12$ jusqu'à l'abattage. Un antibiotique (tartrate de tylosine) a été distribué dans l'eau de boisson pendant les 3 premiers jours et pendant une journée au cours de la quatrième semaine, en vue de prévenir les problèmes de maladie respiratoire. Les aliments utilisés, démarrage jusqu'à 3 semaines d'âge et croissance au-delà, correspondaient à des formules souvent utilisées dans la production non intensive de poulets traditionnels. Leurs principales caractéristiques sont indiquées dans le tableau 1. Ils contenaient un anticoccidien mais pas d'antibiotique et étaient présentés sous forme de farine. 
TableaU 1

Caractéristiques des aliments utilisés, calculées selon les tables INRA (1984).

Calculated characteristics of the diets (according to INRA tables, 1984).

\begin{tabular}{|c|c|c|}
\hline & $\begin{array}{c}\text { Démarrage / Starting diet } \\
(0-3 \text { semaines }) \\
0-3 \text { weeks }\end{array}$ & $\begin{array}{l}\text { Croissance / Growing diet } \\
\text { (après } 3 \text { semaines) } \\
\text { After } 3 \text { weeks }\end{array}$ \\
\hline $\begin{array}{l}\text { Energie métabolisable }(\mathrm{MJ} / \mathrm{kg}) \\
\text { Metabolisable energy }\end{array}$ & 12,37 & 12,34 \\
\hline $\begin{array}{l}\text { Matières azotées totales }(\mathrm{g} / \mathrm{kg}) \quad \ldots \ldots \\
\text { Total crude protein }\end{array}$ & 218 & 196 \\
\hline Lysine $(\mathrm{g} / \mathrm{kg}) \ldots \ldots \ldots$ & 11,4 & 9,9 \\
\hline Méthionine + cystine $(\mathrm{g} / \mathrm{kg})$ & 8,9 & 8,1 \\
\hline Calcium $(g / k g) \ldots \ldots \ldots$ & 10,1 & 9,0 \\
\hline $\begin{array}{l}\text { Phosphore disponible }(\mathrm{g} / \mathrm{kg}) \\
\text { Available phosphorus }\end{array}$ & 4,2 & 3,9 \\
\hline
\end{tabular}

\section{B. Mesures effectuées}

Tous les poulets ont été pesés aux âges de 2,4 , 6 et 7 semaines, après un jeûne complet de 16 heures. L'angle de poitrine a été mesuré sur les animaux vivants à l'âge de 7 semaines à l'aide d'un anglemètre (RICARD, 1961).

Après la pesée de 7 semaines, 20 coquelets et 20 poulettes de chaque lot «densité » ont été prélevés dans les différents fractiles du poids vif de façon à représenter l'ensemble de la population de chaque groupe densité-sexe. Ils ont été abattus dans l'atelier expérimental de la Station, plumés après trempage dans de l'eau à $52{ }^{\circ} \mathrm{C}$, puis effilés (intestins et pancréas enlevés). L'angle de poitrine a été mesuré une nouvelle fois sur les carcasses effilées. Ces dernières ont été enveloppées dans du papier d'aluminium et placées en chambre froide jusqu'au moment de la dissection.

Au cours de la dissection, les pattes ont été sectionnées au niveau de l'articulation tibiotarse-tarsométatarse et la tête à la jonction crâne-atlas. On a pesé séparément la tête, l'ensemble des deux pattes, les dépôts gras abdominaux (situés autour du ventricule succenturié, du gésier et contre la paroi abdominale), le gésier (sans la paroi interne cornée), le foie (sans la vésicule biliaire), le cœur et le cou. Pour se rapprocher de ce qui se passe dans les abattoirs industriels, la moitié de la peau du cou était laissée sur la carcasse. Tous les autres viscères (œsophage, jabot, trachée-artère, poumons, rate, reins, gonades et glande de fabricius) ont été enlevés pour obtenir le poids de la carcasse éviscérée. Sur cette dernière, on a séparé et pesé l'ensemble des muscles pectoraux (c'est-à-dire les muscles situés à l'extérieur de la cage thoracique), les 2 ailes et l'ensemble cuisses + pilons. La dissection a été faite en suivant strictement les points de repère anatomiques, comme l'a proposé le groupe de travail $n^{\circ} 5$ de la W.P.S.A. (1984). Enfin, les cuisses et pilons ont été disséqués pour avoir une estimation de la répartition des tissus : peau, dépôts gras sous-cutanés, os, dépôts gras entre muscles, muscles sans gras (RICARD, 1972 et 1984). 


\section{Calculs réalisés}

L'importance relative des différents éléments de la carcasse a été estimée en calculant différents pourcentages: par rapport au poids vif, au poids de la carcasse éviscérée ou au poids de l'ensemble cuisses + pilons.

Les paramètres statistiques (moyenne, écart-type) ont été calculés pour chaque variable. Les effets densité et sexe ont été testés par les techniques habituelles de l'analyse de variance. De plus, l'influence éventuelle de la vitesse de croissance a été éliminée par analyse de covariance (DAGNelie, 1975).

\section{Résultats}

La mortalité durant l'élevage a été faible : 2 sujets dans le lot à forte densité, 3 dans le lot à faible densité. La différence n'est pas significative $\left(X^{2}=2,2\right)$ et les effectifs sont trop faibles pour qu'on puisse leur attribuer une valeur zootechnique.

Les poids et l'angle de poitrine mesurés sur les poulets vivants figurent dans le tableau 2. Quel que soit le sexe, les valeurs obtenues sont significativement plus élevées dans le lot à densité faible. L'échantillon étudié ne fait pas apparaître de différence significative entre sexes pour l'angle de poitrine. Du fait de la corrélation positive entre poids vif et angle de poitrine $(0,26$ à 0,48 selon les groupes), on peut se demander dans quelle mesure les variations de poids vif peuvent expliquer les variations de l'angle. Les analyses de covariance montrent que même en éliminant l'influence du poids vif, l'angle de poitrine est plus élevé dans le lot à densité faible.

Les valeurs observées pour le poids vif des poulets abattus, l'angle de poitrine mesuré sur la carcasse et le poids des éléments anatomiques de la carcasse sont rassemblés dans le tableau 3 . Les analyses de variance à 2 facteurs, la densité d'élevage et le sexe, n'ont fait apparaitre aucune interaction significative. On peut donc comparer globalement soit les densités, soit les sexes. Ces deux facteurs influencent significativement le poids vif et, par conséquent, de nombreuses variables de dissection : seuls les poids des dépôts adipeux (abdominaux, sous-cutanés ou entre-muscles) ne sont influencés ni par la densité ni par le sexe. Comme pour le poulet vivant, l'angle de poitrine mesuré sur la carcasse est plus élevé dans le lot à densité faible et les différences entre sexes ne sont pas significatives. Le poids des pattes, du foie et du cœur n'est pas influencé par la densité d'élevage.

Les pourcentages, qui mesurent l'importance relative des différents éléments de la carcasse, sont rassemblés dans le tableau 4. Pour ces variables, également, aucune interaction densité $\times$ sexe n'est significative. Les poulets du lot à forte densité ont tendance à avoir un pourcentage plus élevé de dépôts adipeux mais la différence n'est significative que pour les dépôts entre muscles des cuisses et pilons. Les poulets du lot à forte densité présentent des pourcentages significativement plus élevés pour les pattes, le foie et les ailes, mais un pourcentage plus faible pour les muscles des cuisses et pilons. Ils ont aussi des pourcentages plus faibles pour la carcasse éviscérée et les muscles pectoraux mais les différences approchent seulement le seuil de signification. Toutefois, quand on regroupe l'ensemble des muscles disséqués (poitrine + cuisses 
+ pilons) les poulets du lot à forte densité présentent un rendement significativement plus faible que ceux du lot à faible densité, respectivement environ 44 et 45 p. 100 du poids de la carcasse éviscérée.

La comparaison des sexes montre que les poulettes ont des dépôts adipeux plus importants et une plus forte proportion de muscles pectoraux que les coquelets. Elles présentent des pourcentages moins élevés pour les pattes, la tête, le cœur, les membres (ailes, cuisses et pilons) et moins d'os au niveau des cuisses et pilons.

\section{TABLEAU 2}

Résultats des analyses de la vitesse de croissance et de l'angle de poitrine pour l'ensemble des animaux vivants élevés en densité forte (23 poulets au mètre carré) ou faible (10 poulets). Les écarts-types sont indiqués entre parenthèses sous les moyennes.

Comparisons of growth rates and breast angles for all living chickens reared with high or low stocking densities (23 vs 10 birds per square meter).

Standards deviations are in brackets.

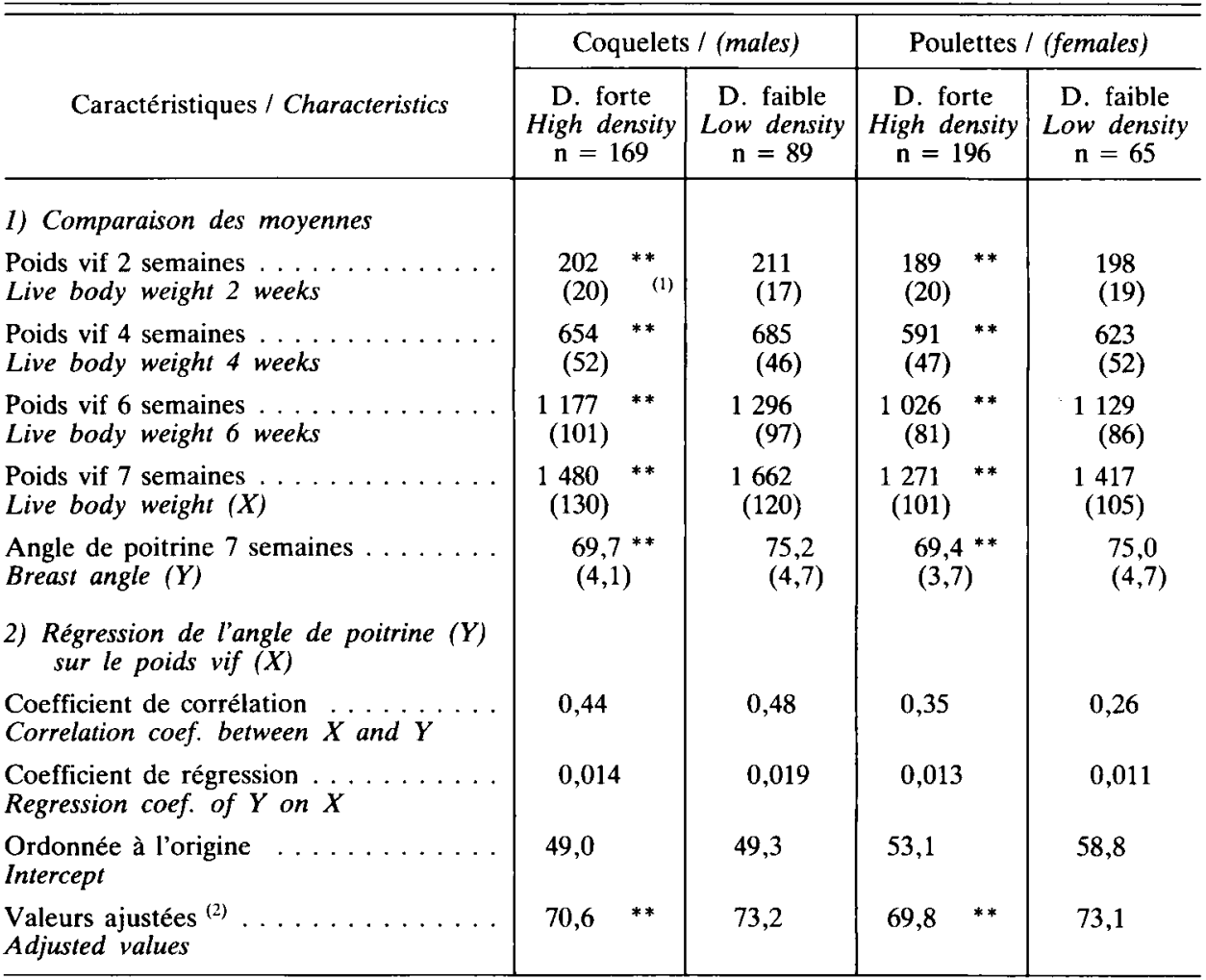

(1) **: Différence significative $(P<0,01)$ entre les deux densités d'élevage

Significant difference $(P<0.01)$ between the two breeding densities.

(2) Valeurs obtenues par analyse de covariance, le poids vif étant maintenu constant. Pour un sexe donné, les deux droites de régression peuvent être considérées comme parallèles $(P<0,05)$.

Values obtained through analysis of covariance, the live weight being maintained constant. For a given sex, the regression lines may be considered as parallel $(P<0.05)$. 


\section{TABleau 3}

Valeurs brutes observées sur les poulets disséqués (20 poulets par groupe sexe-densité d'élevage). Raw values obtained on dissected chickens (20 birds per group).

\begin{tabular}{|c|c|c|c|c|c|c|c|}
\hline \multirow[b]{2}{*}{$\begin{array}{l}\text { Caractéristiques mesurées }{ }^{(1)} \\
\text { Measured characteristics }\end{array}$} & \multicolumn{2}{|c|}{ Coquelets / Males } & \multicolumn{2}{|c|}{ Poulettes / Females } & \multirow[b]{2}{*}{$\mathrm{s}_{\mathrm{i}}{ }^{(2)}$} & \multicolumn{2}{|c|}{$\begin{array}{c}\text { Analyse } \\
\text { de variance } \\
\text { Analysis of variano }\end{array}$} \\
\hline & $\begin{array}{c}\text { Densité } \\
\text { forte } \\
\text { High } \\
\text { density }\end{array}$ & $\begin{array}{c}\text { Densité } \\
\text { faible } \\
\text { Low } \\
\text { density }\end{array}$ & $\begin{array}{c}\text { Densité } \\
\text { forte } \\
\text { High } \\
\text { density }\end{array}$ & $\begin{array}{c}\text { Densité } \\
\text { faible } \\
\text { Low } \\
\text { density }\end{array}$ & & $\begin{array}{c}\text { Effet } \\
\text { densité } \\
\text { Density } \\
\text { effect }\end{array}$ & $\begin{array}{l}\text { Effet } \\
\text { sexe } \\
\text { Sex } \\
\text { effect }\end{array}$ \\
\hline 1. Poids vif avant abattage & $1448,0 \mathrm{~b}$ & $1600,5 \mathrm{a}$ & $1293,2 \mathrm{c}$ & $1401,9 \mathrm{~b}$ & 133,4 & $* *$ & $* *$ \\
\hline 2. Angle poitrine carcasse & $78,9 \mathrm{~b}$ & $81,6 \mathrm{ab}$ & $79,1 \mathrm{~b}$ & 83,7 a & 4,6 & $* *$ & NS \\
\hline 3. Poids gras abdominal .. & 33,3 a & $33,0 \mathrm{a}$ & $34,6 \mathrm{a}$ & $34,0 \mathrm{a}$ & 10,5 & NS & NS \\
\hline 4. Poids des pattes . . . & $73,9 \mathrm{a}$ & $76,8 \mathrm{a}$ & $56,8 \mathrm{~b}$ & $57,4 \mathrm{~b}$ & 6,2 & NS & $* *$ \\
\hline 5. Poids de la tête ... & $42,6 \mathrm{~b}$ & 47,9 a & $36,0 \mathrm{~d}$ & $38,2 \mathrm{c}$ & 3,2 & $* *$ & $* *$ \\
\hline 6. Poids du gésier . . . . . & $25,9 \mathrm{ab}$ & $28,0 \mathrm{a}$ & $24,0 \mathrm{~b}$ & $25,1 \mathrm{~b}$ & 3,7 & $*$ & $* *$ \\
\hline 7. Poids du foie $\ldots \ldots \ldots$ & $29,6 \mathrm{a}$ & 29,8 a & $25,9 \mathrm{~b}$ & $25,8 \mathrm{~b}$ & 2,9 & NS & $* *$ \\
\hline 8. Poids du coeur .... & 7,1 a & 7,6 a & $5,7 \mathrm{~b}$ & $6,2 \mathrm{~b}$ & 1,2 & NS & $* *$ \\
\hline 9. Poids du cou . & $48,0 \mathrm{~b}$ & $54,9 \mathrm{a}$ & $43,7 \mathrm{c}$ & $48,4 \mathrm{~b}$ & 5,1 & $* *$ & $* *$ \\
\hline arcasse éviscérée & $890,6 \mathrm{~b}$ & 955,5 a & $796,4 \mathrm{c}$ & $865,1 \mathrm{~b}$ & 91,9 & $* *$ & $* *$ \\
\hline uscles pectoraux & $171,2 \mathrm{~b}$ & 196,1 a & $160,3 \mathrm{c}$ & $176,1 \mathrm{~b}$ & 21,0 & $* *$ & $* *$ \\
\hline 12. Poids des ailes $\ldots \ldots \ldots$ & $119,3 \mathrm{~b}$ & $131,0 \mathrm{a}$ & $105,6 \mathrm{~d}$ & $112,4 \mathrm{c}$ & 10,7 & $* *$ & $* *$ \\
\hline $\begin{array}{l}\text { 13. Poids cuisses }+ \text { pilons } \\
\text { dont : }\end{array}$ & $348,2 \mathrm{~b}$ & 392,2 a & $300,2 \mathrm{~d}$ & $325,8 \mathrm{c}$ & 36,3 & $* *$ & $* *$ \\
\hline 14. Peau ........ & $32,6 \mathrm{~b}$ & $35,0 \mathrm{a}$ & $28,6 \mathrm{c}$ & $31,0 \mathrm{bc}$ & 5,1 & $*$ & $* *$ \\
\hline 15. Gras sous-cutané . . & $12,2 \mathrm{a}$ & $12,0 \mathrm{a}$ & $12,4 \mathrm{a}$ & 13,3 a & 4,1 & NS & NS \\
\hline 16. Os $\ldots \ldots \ldots$ & $53,1 \mathrm{~b}$ & $60,4 a$ & $42,8 \mathrm{c}$ & $46,1 \mathrm{c}$ & 5,4 & $* *$ & $* *$ \\
\hline 17. Muscles (sans gras) & $219,2 \mathrm{~b}$ & 252,4 a & $190,3 \mathrm{c}$ & $208,0 \mathrm{~b}$ & 23,6 & ** & ** \\
\hline 18. Gras entre-muscles & $7,7 \mathrm{a}$ & $7,7 \mathrm{a}$ & $7,8 \mathrm{a}$ & $7,8 \mathrm{a}$ & 1,7 & NS & NS \\
\hline
\end{tabular}

(1) Les poids sont exprimés en grammes, l'angle de poitrine en grades.

(2) $\mathrm{s}_{\mathrm{i}}$ : écart-type intra-groupe.

(3) Signification des tests $F$ de l'analyse de variance : NS $=$ effet non significatif $(P>0,05),{ }^{*}=$ effet significatif $(P<0,05),{ }^{* *}=$ effet hautement significatif $(P<0,01)$. Aucune intéraction densité $x$ sexe n'est significative.

a, b, c, d : Comparaison multiple des moyennes selon le test de Newmann et Keuls. Deux valeurs suivies de la même lettre ne sont pas statistiquement différentes $(P>0,05)$. La lettre a correspond à la valeur la plus élevée.

English terms: $1=$ live weight before slaughter, $2=$ carcass breast angle, $3=$ abdominal fat weight, $4=$ shanks + feet, $5=$ head, $6=$ gizzard, $7=$ liver, $8=$ heart, $9=$ neck, $i 0=$ eviscerated carcass, $11=$ pectoral muscles, $12=$ wings, $13=$ thighs + drumsticks, $14=$ skin part of thighs + drumsticks, $15=$ subcutaneous fat, $16=$ bones, $17=$ muscles, $18=$ intermuscular fat. 


\section{TABLEAU 4}

Rendements, exprimés en pourcentages, par groupe sexe-densité d'élevage

Yields expressed as percent, for each sex-density group

\begin{tabular}{|c|c|c|c|c|c|c|c|}
\hline \multirow[b]{2}{*}{ Caractéristiques / Characteristics } & \multicolumn{2}{|c|}{ Coquelets / Males } & \multicolumn{2}{|c|}{ Poulettes / Females } & \multirow[b]{2}{*}{$\mathbf{s}_{\mathrm{i}}$} & \multicolumn{2}{|c|}{$\begin{array}{c}\text { Analyse } \\
\text { de variance } \\
\text { Analysis of variance }\end{array}$} \\
\hline & $\begin{array}{l}\text { Densité } \\
\text { forte } \\
\text { High } \\
\text { density }\end{array}$ & $\begin{array}{l}\text { Densité } \\
\text { faible } \\
\text { Low } \\
\text { density }\end{array}$ & $\begin{array}{l}\text { Densité } \\
\text { forte } \\
\text { High } \\
\text { density }\end{array}$ & $\begin{array}{l}\text { Densité } \\
\text { faible } \\
\text { Low } \\
\text { density }\end{array}$ & & $\begin{array}{c}\text { Effet } \\
\text { densité } \\
\text { Density } \\
\text { effect }\end{array}$ & $\begin{array}{l}\text { Effet } \\
\text { sexe } \\
\text { Sex } \\
\text { effect }\end{array}$ \\
\hline \multicolumn{8}{|l|}{$\begin{array}{l}\text { 1) En p. } 100 \text { du poids vif avant } \\
\text { abattage } \\
\text { As percent of live weight be- } \\
\text { fore slaughter }\end{array}$} \\
\hline $\begin{array}{l}\text { Gras abdominal } \ldots \ldots \ldots \ldots \\
\text { Abdominal fat }\end{array}$ & $2,26 \mathrm{ab}$ & $2,04 \mathrm{~b}$ & $2,64 \mathrm{a}$ & $2,42 \mathrm{ab}$ & 0,61 & NS (1) & ** \\
\hline Pattes / Shanks. & 5,12 a & $4,80 \mathrm{~b}$ & $4,40 \mathrm{c}$ & $4,09 \mathrm{~d}$ & 0,25 & $* *$ & $* *$ \\
\hline Tête / Head ... & $2,96 \mathbf{a}$ & $3,00 \mathrm{~b}$ & $2,79 b$ & $2,73 \mathrm{~b}$ & 0,16 & NS & $* *$ \\
\hline Gésier / Gizzard . . . . . . & $1,80 \mathrm{a}$ & $1,76 \mathrm{a}$ & $1,87 \mathrm{a}$ & $1,79 \mathrm{a}$ & 0,11 & NS & NS \\
\hline Foie / Liver $\ldots \ldots \ldots \ldots$ & 2,05 a & $1,86 \mathrm{~b}$ & $2,01 \mathrm{a}$ & $1,84 \mathbf{b}$ & 0,15 & $* *$ & NS \\
\hline Cœur / Heart . . . . . . . . . . & 0,49 a & $0,48 \mathrm{ab}$ & $0,44 \mathrm{~b}$ & $0,44 \mathrm{~b}$ & 0,05 & NS & $* *$ \\
\hline Cou / Neck . . . . . . . . . . & $3,32 \mathrm{a}$ & 3,44 a & $3,38 \mathrm{a}$ & 3,45 a & 0,26 & NS & NS \\
\hline $\begin{array}{l}\text { Carcasse éviscérée } \ldots \ldots \ldots \\
\text { Eviscerated carcass }\end{array}$ & $61,44 \mathrm{a}$ & 62,15 a & 61,51 a & $61,70 \mathrm{a}$ & 1,17 & NS ${ }^{(1)}$ & NS \\
\hline \multicolumn{8}{|l|}{$\begin{array}{l}\text { 2) En p. } 100 \text { du poids de la } \\
\text { carcasse éviscérée } \\
\text { As percent of eviscerated } \\
\text { carcass weight }\end{array}$} \\
\hline $\begin{array}{l}\text { Muscles pectoraux } \ldots \ldots \ldots \\
\text { Pectoral muscles }\end{array}$ & $19,17 \mathrm{~b}$ & $19,71 \mathbf{a b}$ & 20,14 a & $20,36 a$ & 1,05 & NS ${ }^{(1)}$ & $* *$ \\
\hline Ailes / Wings . . . . . . . & 13,46 a & $13,18 \mathrm{ab}$ & $13,29 \mathrm{ab}$ & $13,00 \mathrm{~b}$ & 0,47 & $* *$ & NS ${ }^{(1)}$ \\
\hline $\begin{array}{l}\text { Ensemble cuisses }+ \text { pilons } \ldots \\
\text { Thigh }+ \text { drumsticks }\end{array}$ & 39,10 a & 39,40 a & $37,71 \mathrm{~b}$ & $37,65 \mathrm{~b}$ & 0,81 & NS & ** \\
\hline $\begin{array}{l}\text { Muscles cuisses + pilons .... } \\
\text { Thigh + drumsticks muscles }\end{array}$ & $24,59 \mathrm{~b}$ & 25,37 a & $23,93 \mathrm{c}$ & $24,04 \mathrm{c}$ & 0,86 & * & $* *$ \\
\hline Ensemble des muscles $^{(2)} \ldots$ & $43,76 \mathrm{~b}$ & $45,08 \mathrm{a}$ & $44,07 \mathrm{~b}$ & $44,41 \mathrm{ab}$ & 1,35 & $* *$ & NS \\
\hline $\begin{array}{l}\text { 3) En p. } 100 \text { du poids de } \\
\text { l'ensemble cuisses }+ \text { pilons } \\
\text { As percent of thighs }+ \text { drum- } \\
\text { sticks weight }\end{array}$ & & & & & & & \\
\hline Peau / Skin . . . . . . . . . & 9,35 a & 8,90 a & 9,47 a & $9,53 \mathrm{a}$ & 0,89 & NS & NS \\
\hline $\begin{array}{l}\text { Gras sous-cutané } \cdots \cdots \cdots \\
\text { Subcutaneous fat }\end{array}$ & $3,46 \mathrm{~b}$ & $3,04 \mathrm{~b}$ & 4,06 a & $4,09 \mathrm{a}$ & 1,02 & NS & $* *$ \\
\hline Os / Bones . . . . . . . . . . . & $15,32 \mathrm{a}$ & $15,41 \mathrm{a}$ & $14,30 \mathrm{~b}$ & $14,15 \mathrm{~b}$ & 1,00 & NS & $* *$ \\
\hline $\begin{array}{l}\text { Muscles (sans gras) } \ldots \ldots \ldots \\
\text { Muscles (without fat) }\end{array}$ & $62,90 \mathrm{~b}$ & $64,38 \mathrm{a}$ & $63,46 \mathrm{ab}$ & $63,85 \mathrm{ab}$ & 1,78 & $*$ & NS \\
\hline $\begin{array}{l}\text { Gras entre muscles } \ldots \ldots \ldots \\
\text { Intermuscular fat }\end{array}$ & $2,23 \mathrm{~b}$ & $1,96 \mathrm{c}$ & $2,58 \mathrm{a}$ & $2,39 \mathrm{ab}$ & 0,42 & $*$ & ** \\
\hline
\end{tabular}

(1) Valeur de $F$ proche du seuil de signification $(0,05<P<0,10)$. Aucune interaction sexe $\times$ densité d'élevage n'est significative.

Value of $F: 0.05<P<0.10$. No significant interaction sex $\times$ breeding density.

(2) Muscles pectoraux + muscles cuisses + muscles pilons.

Pectoral muscles + thigh muscles + drumsticks muscles. 
L'influence possible de la vitesse de croissance sur les rendements des différents éléments de la carcasse nous a amené à reprendre les comparaisons densité forte $v s$. densité faible à partir d'analyses de covariance, le poids vif (ou de la carcasse, ou de l'ensemble cuisses + pilons) étant maintenu constant. Les résultats sont rassemblés dans le tableau 5, séparément pour chaque sexe. Dans notre échantillon, qui ne comprend que 20 poulets par groupe élémentaire, l'angle de poitrine mesuré sur la carcasse n'est pas influencé par la densité d'élevage. Par contre, les dépôts adipeux abdominaux ainsi que le poids du foie restent significativement plus importants dans le lot à densité forte, aussi bien chez les coquelets que chez les poulettes, lorsque le poids vif est maintenu constant. Pour les autres caractéristiques de rendement, les différences sont le plus

\section{TABleAU 5}

Analyses de covariance : comparaison des valeurs ajustées pour un poids global fixé. Covariance analyses: comparison of adjusted values when total weight is maintained constant.

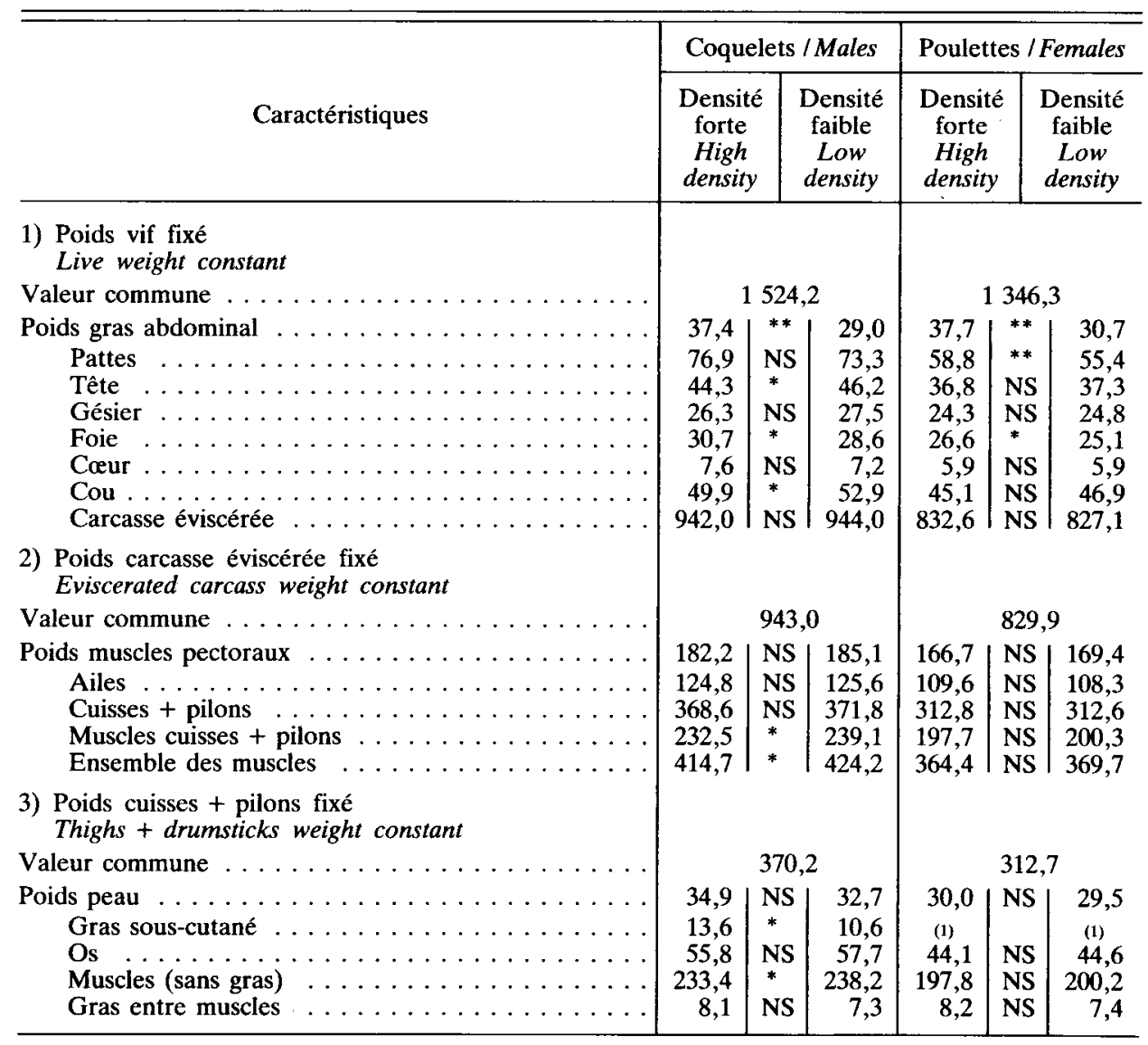

(1) Résultat non indiqué à cause du non parallélisme des droites de régression. Result not given because the regression lines are not parallel. 
souvent non significatives. Chez les coquelets, la tête, le cou et les muscles des cuisses et pilons sont moins importants dans le lot à densité forte tandis que les dépôts adipeux des cuisses et pilons sont plus importants. Chez les poulettes, le poids des pattes reste significativement plus élevé dans le lot à densité forte, quand le poids vif est maintenu constant, alors que pour les coquelets, la différence est dans le même sens mais n'est pas significative.

\section{Discussion}

Les différences hautement significatives observées dans la présente expérimentation pour la vitesse de croissance confirment pleinement les nombreux travaux cités dans la littérature. Dans le tableau 6 ont été regroupés les résultats du poids vif obtenus dans plusieurs expérimentations publiées entre 1960 et 1985 et au cours desquelles on a comparé des densités voisines de 10 et de 20 poulets au mètre carré, pour un élevage au sol. Dans tous les cas, les densités fortes entraînent des poids vifs notablement plus faibles. En contrepartie, l'éleveur augmente nettement le poids de viande produit par unité de surface de poussinière. Dans notre cas, on obtient respectivement 499 et $240 \mathrm{~kg}$ de poulets à l'âge de 7 semaines pour $16 \mathrm{~m}^{2}$ de poussinière (Cf. Tabl. 2).

\section{TABLEAU 6}

Poids vifs moyens (coquelets + poulettes) observés dans 10 expériences où on a comparé un lot à densité forte et un lot à densité faible.

Average body weights (both sexes) in 10 experiments where high or low stocking densities were compared.

\begin{tabular}{|c|c|c|c|c|c|}
\hline & \multirow{2}{*}{ Densités ${ }^{(1)}$} & \multirow{2}{*}{ Age $^{(2)}$} & \multicolumn{3}{|c|}{ Poids vif / Live weight } \\
\hline & & & $\begin{array}{l}\text { Densité } \\
\text { forte }\end{array}$ & $\begin{array}{l}\text { Densité } \\
\text { faible }\end{array}$ & Rapport \\
\hline HANSEN et BECKER (1960) & 22 vs 9 & 11 & 1440 & 1513 & 0,95 \\
\hline MORENG et al. (1961) . . . & 22 vs 9 & 11 & 1288 & 1424 & 0,90 \\
\hline DEATON et al. (1968) . . . . & 22 vs 11 & 8 & 1473 & 1523 & 0,97 \\
\hline BucKLAND et al. (1971) . . & 22 vs 11 & 7 & 1553 & 1610 & 0,96 \\
\hline Bolton et al. (1972) . . . . . & 21 vs 11 & 8 & 1562 & 1568 & 0,996 \\
\hline TSONKOv et BEREMSKI (1975) & 17 vs 9 & 8 & 1567 & 1681 & 0,93 \\
\hline LOPEZ et EscalanTE (1982) & 18 vs 12 & 8 & 1287 & 1358 & 0,95 \\
\hline Polanco et LoPEz (1984) . . . . & 24 vs 14 & 8 & 1492 & 1586 & 0,94 \\
\hline Proudfoot et Hulan (1985) $\ldots$ & 22 vs 12 & 6 & 1693 & 1771 & 0,96 \\
\hline Notre étude /Our study . . . & 23 vs 10 & 7 & 1376 & 1540 & 0,89 \\
\hline
\end{tabular}

(1) Nombre de poulets (sexes mélangés) par mètre carré.

Number of birds (mixed sexes) per square meter.

(2) Age de la pesée, en semaines.

Age (in weeks). 
La présente expérimentation montre que les carcasses obtenues en densité faible sont de meilleure qualité pour un nombre important de caractéristiques anatomiques. L'angle de poitrine plus élevé est à rapprocher du meilleur pourcentage de carcasses de classe A obtenu dans les travaux de Moreng et al. (1961), de Buckland et al. (1971) ou de Proudfoot (1973). La plus forte proportion de dépôts adipeux que nous avons observée en densité forte confirment les résultats de Vo et FanguY (1982). On peut donc obtenir une différence dans l'état d'engraissement en jouant uniquement sur la densité d'élevage. Nous avons obtenu un meilleur rendement à l'abattage (pourcentage de carcasse éviscérée) avec une densité faible mais cette différence n'était pas significative, comme dans les travaux de Proudfoot (1973) et de Scholtyssek (1973). De même, le meilleur pourcentage de muscles pectoraux que nous avons observé confirme les résultats de ScHOLTYSSEK (1973).

Les résultats concernant les caractéristiques de carcasse sont intéressants pour les producteurs de poulets vendus sous " label rouge " et renforcent l'intérêt du règlement français qui limite à 10 poulets au mètre carré la densité d'élevage pour ce type de production (bien que le type génétique et l'âge d'abattage soient différents dans le présent essai et en production label). On constate également que les poulets du lot à faible densité ont une meilleure aptitude à la découpe puisqu'ils ont tendance à avoir moins de dépôts adipeux, un meilleur rendement à l'abattage et un pourcentage un peu plus fort de muscles sur leur carcasse. Mais ces différences ne sont pas suffisantes pour abandonner les densités élevées dans la production industrielle du poulet standard.

Les résultats que nous avons présentés ont été obtenus au cours d'un seul essai. On peut se demander dans quelle mesure ils peuvent être généralisés. Ainsi, notre lot à forte densité donne un poids vif en diminution de 11 p. 100 par rapport au lot à faible densité alors que les résultats cités au tableau 6 correspondent à une diminution moyenne de 5 p. 100 seulement. Il serait intéressant d'étendre ce genre d'expérimentation à d'autres types de poulets (par exemple les souches «label ") et à des formules alimentaires plus riches en énergie si on voulait approfondir les problèmes d'interactions entre génotype, alimentation et densité d'élevage. Mais les données (limitées) de BUCKLAND et al. (1971) ne font pas apparaitre d'interaction significative entre souche et densité.

Reçu en mars 1988.

Accepté en juin 1988.

\section{Remerciements}

La présente expérimentation a pu être réalisée grâce à l'aide financière du Comité Permanent de la Recherche Agronomique de la C.E.E., projet Agrofood F-1-1001. 


\section{Summary \\ Influence of stocking density on growth rate and carcass characteristics of floor reared meat type domestic chickens}

Broilers from a Cornish $\times$ White Rock cross were reared on the floor at high or low stocking densities ( 23 and 10 birds per square meter, respectively, with the same feeder space per bird). All other husbandry procedures were the same.

Birds from the high density group showed significantly lower growth rates and breast angles (Table 2). Raw and yield results obtained on dissected birds are given in Tables 3 and 4, respectively. When growth rate was held constant, fatness was higher and meat yield lower in the high density group (Table 5). Chickens from the high density group also showed higher liver and lower neck percentages than the low density group ones.

Comparisons between sexes confirmed that fat deposits are greater in females than in males. Shank + feet, head and thigh and drumstick percentages were higher in males than in females, whereas pectoral muscles percentages were lower.

Key words : Stocking density, sex, domestic chickens, growth rate, breast angle, fatness, anatomical carcass composition.

\section{Références bibliographiques}

Bolton W., Dewar W.A., Jones R.M., 1972. Effect of stocking density on performance of broiler chick. Br. Poultry Sci., 13, 152-157.

Buckland R.B., GaSPERdone H.C., BragG D.B., 1971. Interaction of strain, density and ration with two light systems on broiler performance. Can J. Anim. Sci., 51, 613-619.

Chrappa V., Peter V., Zila T., Huba J., Bystricky I., 1982. The effect of different stocking rates of broilers in cages on broiler performance. Zivosc. Vyr., 27, 385-390.

Dagnelie P., 1975. Théorie et méthodes statistiques. Vol. 2 : Les Méthodes de l'Inférence Statistique, 341-358. Presses agronomiques, Gembloux (Belgique).

Deaton J.W., Reece F.N., Vardaman T.H., 1968. The effect of temperature and density on broiler performance. Poultry Sci., 47, 293-300.

Hansen R.S., Becker W.A., 1960. Feeding space, population density and growth of young chickens. Poultry Sci., 39, 654-661.

INRA, 1984. L'Alimentation des Animaux Monogastriques, 163-239, Editions INRA, Paris (France).

Lopez S., Escalante A., 1982. Behavior of B-6 broiler according to rearing density by square meter of floor area. Rev. Cubana Cienc. Avic., 9, 153-159.

Moreng R.E., Enos H.L., Buss E.G., Hartung T.E., 1961. The relationship of floor space to factors influencing broiler growth. Poultry Sci., 40, 1039-1044.

Polanco G., Lopez S., 1984. Growth of floor raised broilers using different densities. Rev. Cubana Cienc. Avic., 11, 67-76.

Proudfoot F.G., 1973. Response of broilers to variations in waterer, feeder and floor space under continuous and intermittent photoperiods. Can. J. Anim. Sci., 53, 349-354.

Proudfoot F.G., Hulan H.W., 1985. Effects of stocking density on the incidence of scabby hip syndrome among broiler chickens. Poultry Sci., 64, 2001-2003.

Ricard F.H., 1961. Note sur les méthodes d'estimation de l'angle de poitrine des poulets de chair. Ann. Zootech., 10, 69-72. 
Ricard F.H., 1972. Etude de la composition anatomique du poulet. IV. Possibilités d'estimation des pourcentages de viande, d'os et de peau à partir d'une dissection simplifiée des membres. Ann. Zootech., 21, 49-57.

RicaRd F.H., 1984. Relations entre différents dépôts gras et éléments de peau chez le poulet. Ann. Zootech., 33, 149-160.

Ricard F.H., Touraille C., Marche G., 1986. Influence des méthodes d'élevage sur la qualité des carcasses du poulet. Comptes rendus, $\mathcal{7}^{\mathfrak{C}}$ Conf. Europ. Avic., Larbier M. ed., août 1986, Paris, Vol. 2, 870-873.

Scholtyssex S., 1973. Der Einfluss des Haltungssystems auf das Ausschlachtungsergebnis von Broilern. Arch. Geflügelk., 37, 213-219.

Scholtyssek S., Gschwindt-Ensinger B., 1983. Leistungsvermögen einschliesslich Befiederung und Belastbarkeit von Broilern bei unterschiedlicher Besatzdichte in Bodenhaltung. Arch. Geflügelk., 47, 3-8.

Stanley V.G., Krueger W.F., 1981. The effect of stocking density on commercial broiler performance. Poultry Sci., 60, 1737-1738.

Tsonkov T., Beremski C., 1975. Raising broilers at various densities segregated by sex. Jivotn. Nauki, 12, 75-80.

Vo K.V., Fanguy R.C., 1982. Rearing density as a stressor in the production of commercial broilers. Poultry Sci., 61, 1563 (Abstr.).

Weaver W.D. Jr., Beane W.L., Cherry J.A., 1982. Effect of light, feeding space, stocking density, and dietary energy on broiler performance. Poultry Sci., 61, 33-37.

W.P.S.A., Working Group 5, 1984. Method of dissection of broiler carcasses and description of parts, 33 pp. Jensen J.F. ed., Pendragon Press, Cambridge (Grande-Bretagne). 